Horizons philosophiques

\title{
Note de lecture : Mort et résurrection de la loi morale, de Michel Morin \\ Michel MORIN, Mort et résurrection de la loi morale, Montréal, Les Éditions Hurtibise HMH, Coll. Constantes, 1997, page 42.
}

\section{Jean-Claude Brès}

Volume 9, numéro 2, printemps 1999

La philosophie à portée de voix

URI : https://id.erudit.org/iderudit/801126ar

DOI : https://doi.org/10.7202/801126ar

Aller au sommaire du numéro

Éditeur(s)

Collège Édouard-Montpetit

ISSN

1181-9227 (imprimé)

1920-2954 (numérique)

Découvrir la revue

Citer ce compte rendu

Brès, J.-C. (1999). Compte rendu de [Note de lecture : Mort et résurrection de la loi morale, de Michel Morin / Michel MORIN, Mort et résurrection de la loi morale, Montréal, Les Éditions Hurtibise HMH, Coll. Constantes, 1997, page 42.] Horizons philosophiques, 9(2), 41-49. https://doi.org/10.7202/801126ar d'utilisation que vous pouvez consulter en ligne. 


\title{
NOTE DE LECTURE : MORT ET RÉSURRECTION DE LA LOI MORALE, DE MICHEL MORIN
}

\begin{abstract}
Entre toutes les différentes expressions qui peuvent rendre une seule de nos pensées, il n'y en a qu'une qui soit la bonne. On ne la rencontre pas toujours en parlant ou en écrivant; il est vrai néanmoins qu'elle existe.
\end{abstract}

La Bruyère

Le livre vise le parler net. Michel Morin nous avait déjà habitués à cette recherche, particulièrement depuis Désert. Cette expression toujours plus adéquate à l'objet, cet étirement du langage qui veut réfléchir le retournement vers soi, serait-ce le style qui advient? Peut-être Mort et Résurrection de la loi morale nous propose-t-il avant tout l'examen d'un style, ce qui suppose évidemment un examen de soi. Michel Morin, à l'instar de Nietzsche, veut "écrire avec son sang". Mais n'y eut-il jamais d'autre écriture? Découvrir son style, se rendre, dans l'expression, de plus en plus perméable à l'objet de sa pensée comme à l'accident qui advient, n'est-ce-pas, peu à peu, à travers le langage qui l'instaure dévoiler la valeur qui supporte son existence? "ll est impossible d'exister en tant qu'être humain sans tenter de se valoriser, de faire apparaître à ses propres yeux et à ceux d'autrui que son existence vaut quelque chose'". L'ouvrage qu'ici nous proposons à la lecture a la prétention d'ouvrir à une écriture de soi de plus en plus ferme et rigoureuse parce que de plus en plus liée à une démarche authentique, à une intériorité découverte.

1. Michel MORIN, Mort et résurrection de la loi morale, Montréal, Les Éditions Hurtibise HMH, Coll. Constantes, 1997, page 42. 


\section{Réécrire la loi morale}

Cet exercice n'est pas strictement rhétorique. L'accès à l'Universel semble aujourd'hui compromis, faute de langage. La loi morale, parce qu'abstraite, paraît triste; répétée intarissablement par des groupes et des Églises qui ont perdu leur style et qui n'entraînent plus les consciences, la loi morale ne montre jamais que son aspect coercitif, étrangleur, castrant. "Quelle est la vertu morale réelle d'un individu qui n'est moral que malgré soi2»? La prédication des collectifs de vertu n'est plus entendue que par des individus atomisés, dédaigneux d'euxmêmes, contrits, au corps bafoué.

Redonner voix à l'universel, tel est le projet langagier de l'ouvrage de Michel Morin, la condition stylistique d'un texte qui affirme inaugurer encore le genre éthique. La loi est incisive, tranchante, accusée; l'écriture veut l'être. La loi morale aujourd'hui ne peut prétendre à l'universalité qu'à la condition de ressurgir de ce qu'elle niait jadis : le corps, le particulier, l'accidentel; l'écriture qui pose la nécessité d'une telle loi ne saurait donc être de cette généralité qui encombre et endort dans les manoirs philosophiques. L'exigence d'universel bruit au fond de soi, dans la souffrance qui altère, dans la nuit de soi qui réclame la fin des multiplicités diffuses, dans la déchirure des corps qui fait naître l'Idéal hors des plaintes du désir; l'écriture doit s'acoquiner avec la noirceur, se soumettre aux soubresauts inconscients. Un projet soixante-huitard, un peu fanfaron, un peu romantique, une volonté admirable de ne plus ignorer, dans une boue ratiocinante, les appels de la chair, les cris de la douleur humaine, les égarements de l'individu. Tracé de style qui épouse le sang perdu. Et si de nouveau, la lutte s'organise en soi entre l'appel à l'Idéal et l'écheveau personnel, elle doit conduire, jusque dans la lettre, à l'expression d'un souci de soi qui ne se refuse plus rien, à une sorte de démesure individuelle, de démence du désir. L'exigence la plus haute trouve sa source et son assise en cette coulée de lave. Qui aurait pu croire Agammemnon capable du plus haut sacrifice? Et pourtant ce chef de cité s'il voulait éviter au conflit troyen

2. Ibid., p. 47 
l'enlisement dans la vengeance «ne lui fallait-il pas être capable et avoir le courage de choisir le plus général, mais en même temps le plus exigeant, le plus, à long terme, porteur d'avenir et de justice, fût-ce au prix du sacrifice du plus particulier, représenté à travers le lien toujours étroit entre un père et sa toute jeune fille, incarnation du lien familia|3». À l'égal même des hésitations du héros tragique et des oppositions raisonnées qui le traversent, la phrase s'étire.

Volonté de Michel Morin, tendue dans l'espace de son écrit, de saisir la Parole qui éveille et qui, toujours, est à réentendre. Exercice qui ne peut devenir écriture qu'à la condition de se présenter comme écoute. Sans doute est-ce là le sens réel de ce qui se pressent d'abord à tort comme un commentaire de textes. À lire mieux, c'est d'un surgissement dont il est question à partir des textes, comme si la parole d'Héraclite ou celle de Zarathoustra pouvait encore conduire à penser l'Inespéré; comme si, en nous, Clytemnestre, parfois, poussait son dernier cri. Quand le laisser-aller aux textes conduit la pensée, la navigation est plus sûre. Nous n'imposons pas alors notre petit vouloir, notre paresse de style. Nous attendons des textes qu'à travers leurs rythmes et leurs tracés, le sens s'insinue; "ni spontanéisme irrationnel, ni décision consciente». Une sorte d'entre deux où la Parole est possible, où le texte se monte; entre les pots-pourris des "chanteurs de rue" et la langue mesurée de Créon. L'objectif : trouver la langue, détournée de ses anciens canons, qui enchanterait de nouveau l'appel à l'universel. Après les terribles prophéties de Zarathoustra, la parole sans archipels heurte les récifs tragiques et cherche le souffle du dieu qui, loin de l'horizon des commandements, suggère... À partir d'une sensibilité nouvelle aux commencements qu'une écoute de la voix des antiques héros fait jaillir...

\section{Une phénoménologie de la conscience morale}

Reprendre le chemin de la valorisation mais sur un autre modèle que celui de l'impératif classique. Là où la morale réunissait, le tracé éthique proposé par Michel Morin sépare,

3. Ibid., p. 127 
isole, individualise. Patiemment, au gré des textes où s'exposait jadis la Loi, suivre la démarche qui conduit à retrouver l'idéal. Pas à pas, indiquer l'itinéraire de ce qui, immédiatement, se présente comme conscience. Conscience prise à ras-le-corps dans son attrait vers l'objet; conscience parce qu'il n'est d'autre issue que l'intériorité pour qui désire trouver le sens. "C'est du dedans que le dehors lui arrive". Cette conscience qui se dessine est alors attention aux choses, abandon au mouvement du réel, attrait vers le non-dévoilé, le mal perçu; mais elle est du même coup spontanément distraction; distraction du mémorisé, négation d'elle-même; la conscience-corps souhaite ce qui advient, s'y prépare même, mais sans décision arrêtée. La conscience est invitée à se détourner du représenté, du temps clos et à se mesurer au mouvement même qui est le sien et dont Michel Morin fait l'origine du ressurgissement de la loi morale : l'arrachement. Quand la conscience se fige, elle se leurre, ouvre la porte à l'idéologique, à la formule fétiche, à la Loi étouffante. En lecteur de Nietzsche, Michel Morin suppose que cette fixation a sans doute été une nécessité aussi longtemps que l'humanité n'avait pas accédé à notre degré de civilisation; sans elle, la guerre totale eut peut-être été inévitable; maintenant, l'énoncé dogmatique est signe de mort et qui veut dépasser le nihilisme, danger permanent qui guette la conscience, doit faire place à cette sortie de soi que la conscience effectue quand elle se lie au mouvement du désir. S'arracher, loi du vivant.

De cet arrachement, surgit très vite le souci d'unité. Là où partout le multiple triomphe, une exigence nouvelle se manifeste, impérative à soi, collée au développement de l'intériorité. La conscience-corps veut être autre; autre que la multiple somme de ses déterminations, somme qui enclôt toujours le même. En nous brûle le désir de cet autre désir qui nous fait souffrir. Dans son arrachement à la tribu, à l'enclos national, au sol que le texte aperçoit comme un héritage de Clytemnestre, la conscience s'écarte de l'éparpillement, du chaotique. La méditation d'Antigone est révélatrice en ce sens. L'héroïne consent à se défaire et à mourir non pour obéir à la loi extérieure que le 
pouvoir-Créon aurait formulée mais pour suivre une loi plus universelle, intérieure celle-là, inscrite dans le mouvement qui nous distancie des autres pour mieux nous en approcher. Cette exigence qui se dresse en moi est promesse d'accomplissement notamment par sa permanence : «L'idée, en effet, alors même qu'elle advient au terme d'un effort, d'une tension non seulement de l'esprit mais de l'être tout entier n'est jamais atteinte une fois pour toutes. Elle retombe toujours et à nouveau, il faut en faire l'effort (...) Certes, avec le temps, un [acquis] se constitue mais il perd son sens s'il n'est pas à nouveau et sans cesse réinterprété, plus décisivement encore réinventé, repris et compris de nouveau à la faveur de ce qui dans l'existence est advenu d'autre, de ce qui, parfois imperceptiblement s'est déplacé4»"

Désaisie des choses, tendue vers l'altérité, la conscience est reconnaissance de cette ferveur intérieure qui unifie. La loi, d'une certaine manière, vient de nous, correspond à ce que nous visons de plus élevé, se manifeste enfin par un refus constant des codes et des immobilismes sociaux. Ce n'est que par ce refus que j'entre en contact avec autrui, que ma résistance à me soumettre le rend, à son tour, obligé vis-à-vis de sa propre aspiration à s'élever. La volonté de soi est contagieuse. Hémon aime Antigone. Ici, en lieu et place des coercitions que les lois civiles imposent, Michel Morin suggère l'émulation.

À la tyrannie de Créon, il est naturel de préférer l'humble soumission aimante de l'héroïne Antigone. Serait-ce à dire que le choix moral se fait progressivement entre la tyrannie consentie et l'héroïsme solitaire? L'attachement que l'auteur montre au débat fructueux Créon/Antigone conduit à le penser. Tandis que le pouvoir ouvre sur le vide, la consistance trouve ses racines dans le refus des règles dogmatiques, dans la propension à l'oblatif. Antigone mime le sacrificiel; elle est la proie consentante de ce qui la dépasse. "Clytemnestre eût pu être à la hauteur et consentir au "sacrifice» d'Iphigénie au nom du (...) salut de la Grèce, c'est-à-dire le prestige de l'État, et par delà ce

4. Ibid., p. 124

5. Ibid., p. 134 
dernier l'autorité de la loi et de la justice5»; tandis qu'Iphigénie, elle, dans le texte d'Euripide (Iphigénie à Aulis) mérite toute louange car "en figure inversée de la Mère indigne, elle consent à son propre sacrifice au nom d'un sens de la vie qui va au-delà de sa simple reproduction et de l'attachement de chacun à sa propre survie6». Trop nombreux sont les passages du texte où se développe un lyrisme de l'offrande et de l'oblation comme mode d'accès à la parole paternelle pour que l'on n'y voit qu'une simple indication passagère. De soi, quand la Loi surgit, elle est cruelle, pousse au détachement, à l'arrachement souffrant. Nous retrouvons Zarathoustra. Nous avons donc le choix entre une souffrance mortifère, issue du ressentiment, dont la Mère-nourrice maintiendrait la vigueur, et une souffrance oblative qui nous disposerait à la Parole, qui nous ferait retrouver Raison; l'universalité propre à la Raison consistant cette fois non en des maximes qui accompagneraient nos actions mais dans ce souci de se parfaire et d'accomplir ce qui alors se note destin. Universalité toujours à repenser, consentement à l'Être par un désistement progressif du «je» symptôme de notre modernité, laquelle conduit en effet souvent à l'expression de l'asservissement des individus par des petits "je-Créon» autoritaires ou tyranniques.

La conscience est donc peu à peu conduite à reconnaître en soi la présence de la Loi comme altérité radicale en laquelle elle se plie et se dépasse.

\section{La résurrection du fils}

La loi morale n'est donc possible que si l'on change de génération et que l'on prend Oreste pour modèle au lieu de vouloir à tout prix faire revivre Agammemnon. L'histoire du Fils passe justement par le sacrifice, le meurtre de la Mère, le consentement à la mort du Père. La loi nouvelle exige une régénération. L'engendrement du Fils sauvé, Michel Morin en trouve la matrice dans l'acte de naissance d'Athéna; l'image est grecque, en tous les sens. La Mère a disparu de cet accouchement, le Père est prolongé par un Fils qui était son mal de tête.

6. Ibid., p. 135 
Le Fils ainsi serait débarrassé du ressentiment, lié à l'attachement au sol, à la famille, au donné, au maternel. Oreste aurait, selon l'auteur, ainsi tué ce qu'il y avait de vengeur dans l'antique moralité. Analyse foudroyante de l'ancienne tragédie; pourtant le retour d'Oreste en sa terre, posé dans ces conditions, ne risque-t-il pas de paraître lui aussi comme un geste vengeur? Le dépassement de la loi du sang vaut pour le maternel comme pour le paternel. Oreste retrouve "sa terre", certes ensanglantée, mais désormais prête à recevoir les ondées des Parques. II faut bien que la parole trouve en face d'elle une «Persuasion» et un amour qui l'accueille. Cette vertu n'est-elle pas aussi maternelle?

Les nouvelles tables passent ici par le devenir de I' «individu». Que l'on comprenne bien en quel sens l'auteur retient cette notion; pas question de reproduire un fantôche individualiste mais un "pour-soi», une singularité consciente d'elle-même. Le Fils est seul, héroïque, marqué par le sacrificiel dont il est issu. Michel Morin présente l'individu comme l'accomplissement des Temps Modernes; longtemps, il avait été impossible de penser la morale hors de la conduite collective des moeurs; aujourd'hui, la crise des valeurs a pour effet que nul ne saurait penser une éthique sans qu'elle épouse aussi les tracés individuels de nos destins; que serait une loi qui tout entière échapperait à nos existences? L'individu devient donc celui par lequel la Loi atteint la familiarité des jours.

Lire le texte de Michel Morin, c'est donc en définitive suivre le lent tracé d'une nouvelle filiation. Quand le Fils triomphe du multiple, de la décadence des derniers des hommes, il forge un Nouveau Testament qui appelle un Père autre. Par le sacrificiel, le texte introduit le religieux. Oreste un peu Christ réinvente la Paternité en renonçant à l'antique Loi et, par violence, fonde le sens. Maintenant aucune exigence sinon cet impératif désir de soi, cet engendrement constant dans le tout autre. Le retournement de la violence est dialectique. La mort parentale a rendu vie au Père (mais aussi sans doute à la Mère, délivrée de ses atours trop pesants). L'individu naîtrait de cette longue agonie filiale dont les Atrides fourniraient allégorie. L'individu affirmerait 
le nouvel âge de l'universel qui prend évidemment le masque du tragique. L'engendrement de la Loi passe par le refus des vieilles tables et par la souffrance; au terme de l'ouvrage de Michel Morin, l'Universel surgit nécessairement dans la figure du singulier qu'il brise de toute évidence en raison de l'écartèlement qu'il lui impose. Ce déchirement donne le ton à l'universel dont le caractère abstrait a dès lors été écarté. Qu'on ne s'étonne pas en conséquence que le texte que nous lisons porte le sceau de l'authenticité. Dans un exploit de phénoménologue, Michel Morin ne parle pas la langue officielle des "gargotons" philosophiques; il parle de soi pour tous; ainsi il parle plus net des objets, ainsi il en est plus intègre. Entre Montaigne et Zarathoustra, arqué par une tension intérieure de désir de vérité.

Et on se prend, à la lecture, à ne pas résister à cette invitation à faire soi; comment aujourd'hui pourrait-on se contenter d'une langue kantienne pour traiter de la moralité? Comment, hors de soi, dans une béatitude sommaire, ou à travers une conscience ignorante d'elle-même, pourrions-nous convaincre quiconque de l'intérêt de l'Universel? Pas de loi sans un pour-soi qui la saisisse et la fasse sienne; pas de loi pour ces petits «je» qui prétendent contrôler, imposer leurs règles, ouvrir les avenues du pouvoir dans le retour des refoulés, privés qu'ils sont d'une conscience lucide. Traquer le hasard, volonté débile des petits «je»; faire du hasard nécessité et de cette nécessité acte de volonté, longue méditation spinozienne qui paraît de nouveau chez Michel Morin. Un effort de lucidité est demandé dans le texte pour consentir à voir en soi le dégradé de la Loi, la mort du Père, la place du hasard. Pas d'Unité sans un multiple qui la broie, pas de multiple reconnu sans le consentement intérieur à l'Unité. Le Fils en un sens réconcilie les contraires.

Michel morin écrit et s'écrit. Son écriture invite à une aventure rebelle, celle du Fils qui, dans les sinuosités de l'existence, veut retrouver trace du sens, par lui-même, sans appui. Force de rébellion qui entraîne. Peu à peu l'injonction se brise pour faire place à l'exigence, héroïque parce que solitaire, nuancée parce que mâtinée par chacun des carrefours de l'existence. 
Le «pour-soi» est ici posé comme essence de la moralité. Projet incontestablement romantique, à la différence que le moi ici exposé n'est pas le moi hypertrophié et agonique mais la lucidité de l'individu intérieur lucide. Curieusement pourtant, le "pour-autrui», deuxième temps inévitable d'une conscience morale est réduit dans la synthèse finale au "tout autre"; répudié sous la forme des solidarités humaines parce qu'englué dans le tribal et le maternel; refusé sous la forme institutionnelle parce que soumis aux terreurs citadines dont Créon le tyran fournit la règle. Pourtant Antigone fait surgir l'État, Oreste n'est plus coupable en vertu de la Loi nouvelle qui devra réaménager la cité. On reste donc un peu en suspens dans la lecture des pages consacrées aux Atrides. Où se dresseront les tréteaux des Euménides? Quelle Électre préparera le chemin? On se prend à souhaiter une...suite, une jeu d'alliances qui ferait ressusciter l'appel à la justice à partir d'une vivante remise en cause des solidarités concrètes; à en rester à un "pour-soi", aussi intègre soit-il, ne risque-t-on pas l'enlisement dans la stricte singularité des héros? Mais peut-être ce souhait est-il déjà en retrait de l'ouvrage, une tout autre histoire.

Jean-Claude Brès

Collège Édouard-Montpetit 